\title{
Existence of Solutions for some Quasiaffine PDEs
}

\author{
Flavia Giannetti and Giovanni Pisante
}

Abstract. We prove the existence of solutions of problems of the type

$$
\left\{\begin{aligned}
\Phi(D u(x)) & =f(x) & & \text { in } \Omega \\
u(x) & =\xi_{0} x & & \text { on } \partial \Omega
\end{aligned}\right.
$$

with $\Phi: \mathbb{R}^{n \times n} \rightarrow \mathbb{R}$ quasiaffine function and $\xi_{0} \in \mathbb{R}^{n \times n}$ fixed.

Keywords. Quasiaffine functions, change of variables, multiplication algebra

Mathematics Subject Classification (2000). 35G30, 35A05

\section{Introduction}

In 1965, J. Moser proved that two positive $C^{\infty}$ volume forms on a compact manifold with the same total mass are related by a diffeomorphism [7]. Later on B. Dacorogna and J. Moser in [5] considered problems of the type

$$
\left\{\begin{array}{rlrl}
\operatorname{det} D u(x) & =f & & \text { in } \Omega \\
u(x)=x & & \text { on } \partial \Omega,
\end{array}\right.
$$

where $\Omega$ is a bounded domain in $\mathbb{R}^{n}$ with regular boundary and $f$ is a positive function on $\Omega$ verifying the assumption

$$
\int_{\Omega} f d x=|\Omega|
$$

where $|\Omega|$ denotes the Lebesgue measure of $\Omega$. The equation in (1) is called the prescribed volume form equation and has a lot of applications in physics, in particular in elasticity theory.

F. Giannetti: Dipartimento di Matematica e Applicazioni "R. Caccioppoli", Università di Napoli "Federico II", via Cintia - 80126 Napoli; giannett@unina.it

G. Pisante : Dipartimento di Matematica, Seconda Università degli Studi di Napoli, via Vivaldi 43 - 81100 Caserta; giovanni.pisante@unina2.it 
The main difficulty in solving (1) is in the non-linearity of the Jacobian determinant. In [5], Dacorogna and Moser overcame this problem in the case $f$ belonging to the Hölder spaces $C^{m, \alpha}(\bar{\Omega})$ and obtained the best regularity that one can expect, that is $u \in C^{m+1, \alpha}\left(\bar{\Omega}, \mathbb{R}^{n}\right)$. Later on, in [10], the author obtained the best regularity for $u$ in the case $f$ belonging to the Sobolev space $W^{m, p}(\Omega)$ with $\max \left(1, \frac{n}{m}\right)<p<\infty$, that is $u \in W^{m+1, p}\left(\Omega, \mathbb{R}^{n}\right)$.

The intent of this paper is to continue this theme from a more general and unifying perspective. In particular we study problems of the type

$$
\left\{\begin{aligned}
\Phi(D u(x)) & =f(x) & & \text { in } \Omega \\
u(x) & =\xi_{0} x & & \text { on } \partial \Omega,
\end{aligned}\right.
$$

where $\Phi: \mathbb{R}^{n \times n} \rightarrow \mathbb{R}$ is a quasiaffine function and $\xi_{0} \in \mathbb{R}^{n \times n}$ is fixed. Clearly, in this case we shall require that the function $f$ satisfies the compatibility condition

$$
\int_{\Omega} f(x) d x=\Phi\left(\xi_{0}\right)|\Omega|
$$

Similar arguments to those used in [5] allow us to obtain, for $f \in C^{m, \alpha}(\bar{\Omega})$ and $\left\|f-\Phi\left(\xi_{0}\right)\right\|_{C^{0, \alpha}}$ sufficiently small, a solution in $C^{m+1, \alpha}\left(\bar{\Omega}, \mathbb{R}^{n}\right)$ and an analogous result in the setting of Sobolev spaces. The main difference between our results and the ones contained in [5] and [10] is in the linearization procedure.

\section{Notation and preliminaries}

In this section we introduce some notation and recall some results useful in the sequel. Let $D \subset \mathbb{R}^{n}, u: D \rightarrow \mathbb{R}$ and $0<\alpha \leq 1$ and say

$$
[u]_{\alpha, D}:=\sup _{\substack{x, y \in D \\ x \neq y}}\left\{\frac{|u(x)-u(y)|}{|x-y|^{\alpha}}\right\} .
$$

Let $\Omega \subset \mathbb{R}^{n}$ be open, $k \geq 0$ be an integer. Set $\mathcal{A}_{k}:=\left\{a=\left(a_{1}, \ldots, a_{n}\right) \in \mathbb{N}^{n}\right.$ : $\left.\sum_{j=1}^{n} a_{j}=k\right\}$. An element of $\mathcal{A}_{k}$ is called a multi-index of order $k$. Moreover, if we write $|a|=\sum_{j=1}^{n} a_{j}$, we mean by $D^{a} u$ the derivative $D^{a} u=\frac{\partial^{|a|}}{\partial^{a} x_{1} \cdots \partial^{a_{n}} x_{n}}$.

Define the different spaces of Hölder continuous functions in the following way:

- $C^{0, \alpha}(\Omega)$ is the set of $u \in C(\Omega)$ such that $[u]_{\alpha, K}<\infty$ for every compact set $K \subset \Omega$.

- $C^{0, \alpha}(\bar{\Omega})$ is the set of functions $u \in C(\bar{\Omega})$ such that $[u]_{\alpha, \bar{\Omega}}<\infty$. It is a complete space when equipped with the norm

$$
\|u\|_{C^{0, \alpha}(\bar{\Omega})}:=\|u\|_{C^{0}(\bar{\Omega})}+[u]_{\alpha, \bar{\Omega}} .
$$


- $C^{k, \alpha}(\Omega)$ is the set of $u \in C^{k}(\Omega)$ such that $\left[D^{a} u\right]_{\alpha, K}<\infty$ for every compact set $K \subset \Omega$ and every multi-index $a \in \mathcal{A}_{k}$.

- $C^{k, \alpha}(\bar{\Omega})$ is the set of $u \in C^{k}(\bar{\Omega})$ such that $\left[D^{a} u\right]_{\alpha, \bar{\Omega}}<\infty$ for every multiindex $a \in \mathcal{A}_{k}$. It is a Banach space equipped with the norm

$$
\|u\|_{C^{k, \alpha}}:=\|u\|_{C^{k}}+\max _{a}\left[D^{a} u\right]_{\alpha} .
$$

In the next proposition we state the useful property of Hölder spaces $C^{k, \alpha}(\bar{\Omega})$ of being a multiplication algebra. For further references on these spaces we refer to [1] and [6].

Proposition 2.1. Let $\Omega \subset \mathbb{R}^{n}$ be open, $k \geq 0$ an integer and $0 \leq \alpha \leq 1$. If $u, v \in C^{k, \alpha}(\bar{\Omega})$ then $u v \in C^{k, \alpha}(\bar{\Omega})$. More precisely there exists a constant $c>0$ such that

$$
\|u v\|_{C^{k, \alpha}} \leq c\left(\|u\|_{C^{k, \alpha}}\|v\|_{C^{0}}+\|u\|_{C^{0}}\|v\|_{C^{k, \alpha}}\right) \leq 2 c\|u\|_{C^{k, \alpha}}\|v\|_{C^{k, \alpha}} .
$$

We shall need also the lower semicontinuity property of the $\|\cdot\|_{C^{k, \alpha}}$ norm stated in the following

Proposition 2.2. For an integer $k \geq 0$ and $0<\alpha<1$, let $\Omega \subset \mathbb{R}^{n}$ be a bounded open set with $C^{k+1, \alpha}$ boundary. Let $r>0$ and

$$
B_{r}=\left\{u \in C^{k+1, \alpha}(\bar{\Omega}):\|u\|_{C^{k+1, \alpha}(\bar{\Omega})} \leq r\right\} .
$$

Let $\left\{u_{h}\right\}_{h \in \mathbb{N}} \subset B_{r}$ such that $u_{h} \rightarrow u$ in $L^{\infty}(\Omega)$, then $u \in B_{r}$ and

$$
\|u\|_{C^{k+1, \alpha}(\bar{\Omega})} \leq \liminf _{h \rightarrow \infty}\left\|u_{h}\right\|_{C^{k+1, \alpha}(\bar{\Omega})} .
$$

For the proof we suggest the book of Dacorogna ( [3], Prop. 12.8).

The following theorem, fundamental for the proof of our main result, has been investigated by several authors, see for example [4].

Theorem 2.3. Let $k \geq 0$ an integer and $0<\alpha<1$. Let $\Omega \subset \mathbb{R}^{n}$ be a bounded connected open set with orientable $C^{k+3, \alpha}$ boundary consisting of finitely many connected components. The following conditions are then equivalent:

(i) $f \in C^{k, \alpha}(\bar{\Omega})$ satisfies

$$
\int_{\Omega} f(x) d x=0
$$

(ii) There exists $u \in C^{k+1, \alpha}\left(\bar{\Omega}, \mathbb{R}^{n}\right)$ verifying

$$
\left\{\begin{aligned}
\operatorname{div} u=f & \text { in } \Omega \\
u=0 & \text { on } \partial \Omega .
\end{aligned}\right.
$$

Furthermore, there exists $C_{0}=C_{0}(\alpha, k, \Omega)>0$ such that

$$
\|u\|_{C^{k+1, \alpha}} \leq C_{0}\|f\|_{C^{k, \alpha}} .
$$


Let us recall now the definition and some structure properties of quasiaffine functions.

A Borel measurable and locally integrable function $\Phi: \mathbb{R}^{n \times N} \rightarrow \mathbb{R}$ is said to be quasiconvex (in the sense of Morrey) if

$$
\Phi(A) \leq \frac{1}{|D|} \int_{D} \Phi(A+\nabla \phi(x)) d x
$$

for every bounded domain $D \subset \mathbb{R}^{n}$, for every $A \in \mathbb{R}^{n \times N}$ and for every $\phi \in$ $W_{0}^{1, \infty}\left(D ; \mathbb{R}^{N}\right)$.

We say that a Borel measurable and locally integrable function $\Phi: \mathbb{R}^{n \times N} \rightarrow$ $\mathbb{R}$ is quasiaffine if $\Phi$ and $-\Phi$ are quasiconvex. Quasiaffine functions are completely characterized in [2] as linear functions of minors. In order to write the representations of a quasiaffine function let us fix the terminology used.

For a matrix $A \in \mathbb{R}^{n \times N}$ we denote by $T(A)$ the vector

$$
T(A)=\left(A, \operatorname{adj}_{2} A, \ldots, \operatorname{adj}_{n \wedge N} A\right),
$$

where $\operatorname{adj}_{s} A$ stands for the matrix of all $s \times s$ minors of the matrix $A$, with $2 \leq s \leq n \wedge N=\min \{n, N\}$. Observe that $T$ can be seen as an operator $T: \mathbb{R}^{n N} \rightarrow \mathbb{R}^{\tau(n, N)}$ where $\tau(n, N)=\sum_{s=1}^{n \wedge N}\left(\begin{array}{c}N \\ s\end{array}\right)\left(\begin{array}{l}n \\ s\end{array}\right)$ is the euclidean dimension of the space of all minors of $A$.

The following proposition holds true.

Proposition 2.4. Let $\Phi: \mathbb{R}^{n \times N} \rightarrow \mathbb{R}$. Then $\Phi$ is quasiaffine if and only if there exists $\beta \in \mathbb{R}^{\tau(n, N)}$ such that

$$
\Phi(A)=\Phi(0)+\langle\beta ; T(A)\rangle
$$

for every $A \in \mathbb{R}^{n \times N}$, where $\langle\cdot ; \cdot\rangle$ denotes the scalar product in $\mathbb{R}^{\tau(n, N)}$.

We refer to the book [3] and to the references therein for further properties and characterizations of quasiaffine functions.

\section{The equation $\Phi(D u)=f$}

In this section we present our main result in the setting of Hölder spaces. Let $\Phi: \mathbb{R}^{n \times n} \rightarrow \mathbb{R}$ a quasiaffine function and $\xi_{0} \in \mathbb{R}^{n \times n}$ such that $\operatorname{det} D \Phi\left(\xi_{0}\right) \neq 0$. The following result holds:

Theorem 3.1. Let $m \geq 0$ an integer and $0<\alpha<1$. Let $\Omega \subset \mathbb{R}^{n}$ be a bounded connected open set with orientable $C^{m+3, \alpha}$ boundary consisting of finitely many connected components. Assume $f \in C^{m, \alpha}(\bar{\Omega})$ satisfying

$$
\int_{\Omega} f(x) d x=\Phi\left(\xi_{0}\right)|\Omega|
$$


Then there exists $\epsilon=\epsilon(\alpha, m, \Omega)>0$ such that if $\left\|f-\Phi\left(\xi_{0}\right)\right\|_{C^{0, \alpha}(\bar{\Omega})} \leq \epsilon$, then there exists $u \in C^{m+1, \alpha}\left(\bar{\Omega}, \mathbb{R}^{n}\right)$ such that

$$
\left\{\begin{aligned}
\Phi(D u(x)) & =f(x), & & x \in \Omega \\
u(x) & =u_{\xi_{0}}(x):=\xi_{0} x, & & x \in \partial \Omega .
\end{aligned}\right.
$$

Furthermore, there exists a constant $\gamma=\gamma(\alpha, m, \Omega)$ such that

$$
\left\|u-u_{\xi_{0}}\right\|_{C^{1, \alpha}\left(\bar{\Omega}, \mathbb{R}^{n}\right)} \leq \gamma\left\|f-\Phi\left(\xi_{0}\right)\right\|_{C^{0, \alpha}(\bar{\Omega})} .
$$

Remark 3.2. Assuming in addition that det $\xi_{0}>0$, we also have that $u(x)$ is a diffeomorphism provided $\left\|f-\Phi\left(\xi_{0}\right)\right\|_{C^{0, \alpha}(\bar{\Omega})}$ is sufficiently small. Indeed $u_{\xi_{0}}(x)$ is one to one and, in virtue of (3), for $\epsilon$ small enough there exists $\epsilon^{\prime}>0$ such that $\operatorname{det} D u(x) \geq \operatorname{det} \xi_{0}-\epsilon^{\prime}>0$ (see for example [8]).

Proof of Theorem 3.1. We divide the proof into two steps.

Step 1. Setting $v(x)=u(x)-u_{\xi_{0}}(x)$, the problem in (2) can be read as

$$
\left\{\begin{aligned}
\Phi\left(\xi_{0}+D v(x)\right) & =f(x) & & \text { in } \Omega \\
v(x) & =0 & & \text { on } \partial \Omega .
\end{aligned}\right.
$$

On the other hand, since $\Phi$ is an affine function of the minors (see Proposition 2.4), it is a polinomial function of its arguments. It follows that its Taylor expansion has a finite number of entries and then we can write

$$
\Phi\left(\xi_{0}+D v(x)\right)=\Phi\left(\xi_{0}\right)+\left\langle D \Phi\left(\xi_{0}\right) ; D v(x)\right\rangle+Q_{\xi_{0}}(D v(x)),
$$

where $Q_{\xi_{0}}(\zeta)$ is a polinomial function that does not involve any linear terms in the $\zeta$ variable. Therefore the problem (4) can be rewritten as

$$
\left\{\begin{aligned}
\left\langle D \Phi\left(\xi_{0}\right) ; D v(x)\right\rangle+Q_{\xi_{0}}(D v(x)) & =f(x)-\Phi\left(\xi_{0}\right) & & \text { in } \Omega \\
v(x) & =0 & & \text { on } \partial \Omega .
\end{aligned}\right.
$$

Let us show, by using some arguments of linear algebra, that the linear term in (5) can be simplified in a divergence.

Let $H$ be an invertible $n \times n$ matrix and consider the change of variables $y=H x$. Define $w(y)=v\left(H^{-1} y\right)$; its gradient will be given by

$$
D w(y)=D v\left(H^{-1} y\right) H^{-1}=D v(x) H^{-1},
$$

and therefore the gradient of $v$ can be written as $D v(x)=D w(y) H$. The linear term in (5) becomes $\left\langle D \Phi\left(\xi_{0}\right) ; D w(y) H\right\rangle$ or equivalently $\left\langle D \Phi\left(\xi_{0}\right) H^{T} ; D w(y)\right\rangle$. Since det $D \Phi\left(\xi_{0}\right) \neq 0$, we can choose $H:=\left(D \Phi\left(\xi_{0}\right)^{T}\right)^{-1}$ and have

$$
\left\langle D \Phi\left(\xi_{0}\right) H^{T} ; D w(y)\right\rangle=\operatorname{div} w .
$$


Then the problem (5) assumes the form

$$
\left\{\begin{aligned}
\operatorname{div} w & =\tilde{f}-\Phi\left(\xi_{0}\right)-\tilde{Q}_{\xi_{0}}(D w) & & \text { in } H \Omega \\
w & =0 & & \text { on } \partial(H \Omega),
\end{aligned}\right.
$$

where we have defined $\tilde{f}:=f \circ H^{-1}$ and $\tilde{Q}_{\xi_{0}}:=Q_{\xi_{0}} \circ H^{-1}$.

Step 2. Denote by $H \Omega \subset \mathbb{R}^{n}$ the domain obtained by the deformation of the domain $\Omega$ via the linear mapping represented by $H$ and consider the linear spaces

$$
\begin{aligned}
& X=\left\{b \in C^{m, \alpha}(\overline{H \Omega}): \int_{H \Omega} b(x) d x=0\right\} \\
& Y=\left\{a \in C^{m+1, \alpha}\left(\overline{H \Omega}, \mathbb{R}^{n}\right): a=0 \text { on } \partial(H \Omega)\right\} .
\end{aligned}
$$

By Theorem 2.3 we know that there exists a bounded linear operator $L: X \rightarrow Y$ which associates to every $b \in X$ a unique $a \in Y$ such that

$$
\left\{\begin{aligned}
\operatorname{div} a=b & \text { in } H \Omega \\
a=0 & \text { on } \partial(H \Omega) .
\end{aligned}\right.
$$

Furthermore, there exists a constant $C_{0}>0$ such that

$$
\|L b\|_{C^{m+1, \alpha}\left(\overline{H \Omega}, \mathbb{R}^{n}\right)} \leq C_{0}\|b\|_{C^{m, \alpha}(\overline{H \Omega})} .
$$

If we define for $w \in C^{m+1, \alpha}\left(\overline{H \Omega}, \mathbb{R}^{n}\right)$ the operator

$$
N(w)=\tilde{f}-\Phi\left(\xi_{0}\right)-\tilde{Q}_{\xi_{0}}(D w),
$$

we can easily deduce that (6) is solved for any $w \in Y$ which is a fixed point of the operator $L N$, i.e.,

$$
w=L N(w)
$$

Let us remark that the equation (8) is well defined for any $w \in Y$. Indeed, being $\tilde{Q}_{\xi}(\zeta)$ analytic, we obviously have $N(w) \in C^{m, \alpha}$. Moreover, using an integration by parts argument and the structure of $Q_{\xi_{0}}$, we infer that

$$
\int_{H \Omega} N(w) d y=\int_{H \Omega}\left[\tilde{f}-\Phi\left(\xi_{0}\right)-\tilde{Q}_{\xi_{0}}(D w)\right] d y=0
$$

since $w=0$ on $\partial(H \Omega)$ and $\int_{H \Omega}\left[\tilde{f}-\Phi\left(\xi_{0}\right)\right] d y=0$.

Our aim is to use the contraction mapping principle to solve (8). For $r>0$, consider the set

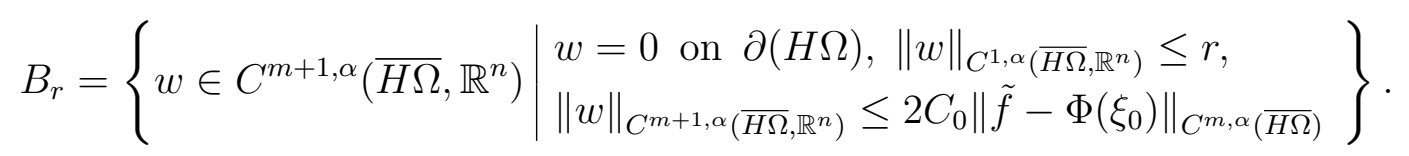


This is a complete space when endowed with the $C^{1, \alpha}$ norm (see Proposition 2.2). We shall prove the existence of $w \in B_{r}$ satisfying (8). First of all, note that there exists a constant $C_{1}>0$ such that

$$
\left\|\tilde{f}-\Phi\left(\xi_{0}\right)\right\|_{C^{0, \alpha}(\overline{H \Omega})} \leq C_{1}\left\|f-\Phi\left(\xi_{0}\right)\right\|_{C^{0, \alpha}(\bar{\Omega})} .
$$

Moreover, from Proposition 2.1 we get the existence of a constant $c>0$ such that if $w_{1}, w_{2} \in C^{m+1, \alpha}\left(\overline{H \Omega}, \mathbb{R}^{n}\right)$ with $\left\|w_{1}\right\|_{C^{1, \alpha}},\left\|w_{2}\right\|_{C^{1, \alpha}} \leq 1$, then

$$
\left\|\tilde{Q}_{\xi_{0}}\left(D w_{1}\right)-\tilde{Q}_{\xi_{0}}\left(D w_{2}\right)\right\|_{C^{0, \alpha}} \leq c\left(\left\|w_{1}\right\|_{C^{1, \alpha}}+\left\|w_{2}\right\|_{C^{1, \alpha}}\right)\left\|w_{1}-w_{2}\right\|_{C^{1, \alpha}} .
$$

Fix $\epsilon=\min \left\{\frac{1}{8 c C_{1} C_{0}^{2}}, \frac{1}{2 C_{0} C_{1}}, \frac{1}{4 c C_{1} C_{0}}\right\}$ and $r=2 C_{0}\left\|\tilde{f}-\Phi\left(\xi_{0}\right)\right\|_{C^{0, \alpha}(\overline{H \Omega})}$. If we note that $r \leq 1$, for $w_{1}, w_{2} \in B_{r}$ we have

$$
\begin{aligned}
\left\|L N\left(w_{1}\right)-L N\left(w_{2}\right)\right\|_{C^{1, \alpha}} & \leq C_{0}\left\|N\left(w_{1}\right)-N\left(w_{2}\right)\right\|_{C^{0, \alpha}} \\
& =C_{0}\left\|\tilde{Q}_{\xi_{0}}\left(D w_{1}\right)-\tilde{Q}_{\xi_{0}}\left(D w_{2}\right)\right\|_{C^{0, \alpha}} \\
& \leq c C_{0}\left(\left\|w_{1}\right\|_{C^{1, \alpha}}+\left\|w_{2}\right\|_{C^{1, \alpha}}\right)\left\|w_{1}-w_{2}\right\|_{C^{1, \alpha}} \\
& \leq 2 r c C_{0}\left\|w_{1}-w_{2}\right\|_{C^{1, \alpha}} \\
& =4 c C_{0}^{2}\left\|\tilde{f}-\Phi\left(\xi_{0}\right)\right\|_{C^{0, \alpha}}\left\|w_{1}-w_{2}\right\|_{C^{1, \alpha}} \\
& \leq 4 c C_{1} C_{0}^{2}\left\|f-\Phi\left(\xi_{0}\right)\right\|_{C^{0, \alpha}}\left\|w_{1}-w_{2}\right\|_{C^{1, \alpha}} \\
& \leq \frac{1}{2}\left\|w_{1}-w_{2}\right\|_{C^{1, \alpha}} .
\end{aligned}
$$

Moreover, observing that for $w \in B_{r}$

$$
\begin{aligned}
\left\|\tilde{Q}_{\xi_{0}}(D w)\right\|_{C^{m, \alpha}} & \leq c\|w\|_{C^{m+1, \alpha}}\|w\|_{C^{1, \alpha}} \\
& \leq 2 c C_{0}\|w\|_{C^{m+1, \alpha}}\left\|\tilde{f}-\Phi\left(\xi_{0}\right)\right\|_{C^{0, \alpha}} \\
& \leq 2 c C_{1} C_{0}\|w\|_{C^{m+1, \alpha}}\left\|f-\Phi\left(\xi_{0}\right)\right\|_{C^{0, \alpha}} \\
& \leq \frac{1}{4 C_{0}}\|w\|_{C^{m+1, \alpha}} \\
& \leq \frac{1}{2}\left\|\tilde{f}-\Phi\left(\xi_{0}\right)\right\|_{C^{m, \alpha}}
\end{aligned}
$$

we get

$$
\begin{aligned}
\|L N(w)\|_{C^{m+1, \alpha}} & \leq C_{0}\|N(w)\|_{C^{m, \alpha}} \\
& \leq C_{0}\left\|\tilde{f}-\Phi\left(\xi_{0}\right)\right\|_{C^{m, \alpha}}+C_{0}\left\|\tilde{Q}_{\xi_{0}}(D w)\right\|_{C^{m, \alpha}} \\
& \leq 2 C_{0}\left\|\tilde{f}-\Phi\left(\xi_{0}\right)\right\|_{C^{m, \alpha}} .
\end{aligned}
$$

Finally,

$$
\begin{aligned}
\|L N(w)\|_{C^{1, \alpha}} & \leq\|L N(w)-L N(0)\|_{C^{1, \alpha}}+\|L N(0)\|_{C^{1, \alpha}} \\
& \leq \frac{1}{2}\|w\|_{C^{1, \alpha}}+C_{0}\|N(0)\|_{C^{0, \alpha}} \\
& \leq \frac{r}{2}+C_{0}\left\|\tilde{f}-\Phi\left(\xi_{0}\right)\right\|_{C^{0, \alpha}}=r .
\end{aligned}
$$


In conclusion $L N: B_{r} \rightarrow B_{r}$ is a contraction mapping, hence there exists a solution $v$ in $C^{m+1, \alpha}$ of the problem (5).

Furthermore, since the function $w$ found above satisfies the inequality

$$
\|w\|_{C^{1, \alpha}} \leq 2 C_{0}\left\|\tilde{f}-\Phi\left(\xi_{0}\right)\right\|_{C^{0, \alpha}}
$$

and there exists a constant $K>0$ such that $\|v\|_{C^{1, \alpha}\left(\bar{\Omega}, \mathbb{R}^{n}\right)} \leq K\|w\|_{C^{1, \alpha}\left(\overline{H \Omega}, \mathbb{R}^{n}\right)}$ we get the existence of $\gamma=\gamma(\alpha, m, \Omega)$ such that $\left\|u-u_{\xi_{0}}\right\|_{C^{1, \alpha}} \leq \gamma\left\|f-\Phi\left(\xi_{0}\right)\right\|_{C^{0, \alpha}}$. Theorem 3.1 is proved.

Remark 3.3. Note that, choosing in the proof of Theorem 3.1 instead of $B_{r}$, the set $B$ defined as

$$
B=\left\{\begin{array}{l|l}
w \in C^{m+1, \alpha}\left(\overline{H \Omega}, \mathbb{R}^{n}\right) & \begin{array}{l}
w=0 \text { on } \partial(H \Omega), \forall k \in\{0, \ldots, m\} \\
\|w\|_{C^{k+1, \alpha}\left(\overline{H \Omega}, \mathbb{R}^{n}\right)} \leq 2 C_{0}\left\|\tilde{f}-\Phi\left(\xi_{0}\right)\right\|_{C^{k, \alpha}(\overline{H \Omega})}
\end{array}
\end{array}\right\}
$$

we can also obtain the estimates

$$
\left\|u-u_{\xi_{0}}\right\|_{C^{k+1, \alpha}\left(\bar{\Omega}, \mathbb{R}^{n}\right)} \leq \gamma\left\|f-\Phi\left(\xi_{0}\right)\right\|_{C^{k, \alpha}(\bar{\Omega})},
$$

just replacing $m$ with $k$ in the previous proof.

\section{The Sobolev case}

In this last section we point how to extend Theorem 3.1 to the setting of Sobolev spaces. We shall start stating the result and then we shall comment about the proof. Let $\Phi: \mathbb{R}^{n \times n} \rightarrow \mathbb{R}$ a quasiaffine function and $\xi_{0} \in \mathbb{R}^{n \times n}$ such that $\operatorname{det} D \Phi\left(\xi_{0}\right) \neq 0$.

Theorem 4.1. Let $m \in \mathbb{N}, p>\frac{n}{m}$ and $0<\alpha \leq m-\frac{n}{p}$. Let $\Omega \subset \mathbb{R}^{n}$ be a bounded connected open set with orientable $C^{m+3}$ boundary consisting of finitely many connected components. Assume $f \in W^{m, p}(\Omega)$ satisfying

$$
\int_{\Omega} f(x) d x=\Phi\left(\xi_{0}\right)|\Omega|
$$

Then there exists $\epsilon=\epsilon(p, m, \Omega)>0$ such that if $\left\|f-\Phi\left(\xi_{0}\right)\right\|_{C^{0, \alpha}(\bar{\Omega})} \leq \epsilon$, then there exists $u \in W^{m+1, p}\left(\Omega, \mathbb{R}^{n}\right)$ such that

$$
\left\{\begin{aligned}
\Phi(D u(x)) & =f(x), & & x \in \Omega \\
u(x) & =u_{\xi_{0}}(x):=\xi_{0} x, & & x \in \partial \Omega .
\end{aligned}\right.
$$


Theorem 4.1 can be seen as the analogous of the result of Ye [10] on the prescribed volume form equation. Since the proof of Theorem 4.1 retraces the one of Theorem 3.1 here we shall just make some comments on the arguments needed to justify all the steps. Step 1 goes exactly as in Theorem 3.1.

Concerning Step 2, we first note that if $p>\frac{n}{m}$, since the regularity of $\partial \Omega$ implies the strong local Lipschitz property, the Sobolev space $W^{m, p}(\Omega)$ is embedded in the Hölder space $C^{k, \beta}(\bar{\Omega})$, with $k:=\max \{j:(m-j) p>n\}$ and $0<\beta<1$ if $(m-k-1) p=n$ or $0<\beta \leq m-k-\frac{n}{p}$ otherwise (see [1]). Accordingly, in our hypotheses, we always have $W^{m, p}(\Omega) \hookrightarrow C^{0, \alpha}(\bar{\Omega})$ and therefore $f \in C^{0, \alpha}(\bar{\Omega})$. This justifies our assumption on the difference $f-\Phi\left(\xi_{0}\right)$.

The existence of the bounded linear operator $L$ can still be proved using elliptic equations arguments (see for example Theorem 2 in [10]). The spaces $X$ and $Y$ are replaced by

$$
\begin{aligned}
& \tilde{X}=\left\{b \in W^{m, p}(H \Omega): \int_{H \Omega} b(x) d x=0\right\} \\
& \tilde{Y}=\left\{a \in W^{m+1, p}\left(H \Omega, \mathbb{R}^{n}\right): a=0 \text { on } \partial(H \Omega)\right\},
\end{aligned}
$$

respectively, and endowed with the Sobolev norms, in such a way that the boundedness of $L$ can be rephrased as

$$
\|L b\|_{W^{m+1, p}\left(H \Omega, \mathbb{R}^{n}\right)} \leq C_{0}\|b\|_{W^{m, p}(H \Omega)} .
$$

We note also that in this framework it is enough to suppose $\partial \Omega$ of class $C^{m+3}$ and that, in virtue of the recalled Sobolev embedding, the condition on the boundary in the problem (7) has a pointwise meaning.

The contraction mapping principle can be applied to the set

$$
\tilde{B}=\left\{\begin{array}{l|l}
w \in W^{m+1, p}\left(H \Omega, \mathbb{R}^{n}\right) & \begin{array}{l}
w=0 \text { on } \partial(H \Omega), \\
\|w\|_{C^{1, \alpha}\left(\overline{H \Omega}, \mathbb{R}^{n}\right)} \leq 2 C_{0}\left\|\tilde{f}-\Phi\left(\xi_{0}\right)\right\|_{C^{0, \alpha}(\overline{H \Omega})}, \\
\|w\|_{W^{m+1, p}\left(H \Omega, \mathbb{R}^{n}\right)} \leq 2 C_{0}\left\|\tilde{f}-\Phi\left(\xi_{0}\right)\right\|_{W^{m, p}(H \Omega)}
\end{array}
\end{array}\right\} .
$$

The completeness of $\tilde{B}$ with respect to the $C^{1, \alpha}$ norm follows from the sequential weak relative compactness of bounded sets in $W^{m+1, p}$, the lower semicontinuity of the $W^{m+1, p}$ norm with respect to the weak convergence, and from the completeness of $C^{1, \alpha}\left(\overline{H \Omega}, \mathbb{R}^{n}\right)$.

Finally, in order to estimate $\left\|\tilde{Q}_{\xi_{0}}\right\|_{W^{m, p}}$, we need the property of $W^{m, p}(H \Omega)$ of being a Banach algebra. This property, when $m p>n$, follows easily from the Sobolev embedding theorems (see [1] for details).

We conclude observing that the choice of $\tilde{B}$ implies for $u$ the estimate

$$
\left\|u-u_{\xi_{0}}\right\|_{W^{m+1, p}\left(\Omega, \mathbb{R}^{n}\right)} \leq \gamma\left\|f-\Phi\left(\xi_{0}\right)\right\|_{W^{m, p}(\Omega)},
$$


and following the same argument used in Remark 3.3 we can estimate the distance from $u$ and $u_{\xi_{0}}$ in any norm which generates a smaller topology with respect to the $W^{m, p}$ one and such that the bounded sets are, for example, sequentially relatively compact with respect to the $L^{\infty}$ convergence. For instance, we can infer the following $W^{k, p}$ estimates for $k<m$ :

$$
\left\|u-u_{\xi_{0}}\right\|_{W^{k+1, p}\left(\Omega, \mathbb{R}^{n}\right)} \leq \gamma\left\|f-\Phi\left(\xi_{0}\right)\right\|_{W^{k, p}(\Omega)} .
$$

\section{References}

[1] Adams, R. A., Sobolev Spaces. Pure and Applied Mathematics, vol. 65, New York: Academic Press 1975.

[2] Ball, J. M., Convexity conditions and existence theorems in nonlinear elasticity. Arch. Rational Mech. Anal. 63 (1976/77)(4), 337 - 403.

[3] Dacorogna, B., Direct Methods in the Calculus of Variations (2nd ed.). New York: Springer 2008.

[4] Dacorogna, B., Existence and regularity of solutions of $d \omega=f$ with Dirichlet boundary conditions. In: Nonlinear Problems in Mathematical Physics and Related Topics I. Int. Math. Ser. (N. Y.) 1. New York: Kluwer/Plenum 2002, pp. $67-82$.

[5] Dacorogna, B. and Moser, J., On a partial differential equation involving the Jacobian determinant. Ann. Inst. H. Poincaré Anal. Non Linéaire 7 (1990), $1-26$.

[6] Gilbarg, D. and Trudinger, N., Elliptic Partial Differential Equations of Second Order (reprint of the 1998 ed.). Classics in Mathematics. Berlin: Springer 2001.

[7] Moser, J., On the volume elements on a manifold. Trans. Amer. Math. Soc. 120 (1965), $286-294$.

[8] Meisters, G. H. and Olech, C., Locally one to one mappings and a classical theorem on schlicht functions. Duke Math. J. 30 (1963), $63-80$.

[9] Riviére, T. and Ye, D., Resolutions of the prescribed volume form equation. Nonlin. Diff. Eqs. Appl. 3 (1996), 323 - 369.

[10] Ye, D., Prescribing the Jacobian determinant in Sobolev spaces. Ann. Inst. H. Poincaré Anal. Non Liné aire 3 (1994), 275 - 296.

Received April 16, 2007 